\title{
NEW POSSIBILITIES OF RADIATION CONTROL OF QUALITY OF WELDED JOINTS
}

\author{
V.A. TROITSKY \\ E.O. Paton Electric Welding Institute, NASU \\ 11 Bozhenko Str., 03680, Kiev, Ukraine. E-mail: office@paton.kiev.ua
}

\begin{abstract}
The digital technologies get ever wider application in various fields of engineering, including also in radiation control of quality of welded joints. In the work a high flaw detection efficiency of flash radiography and radiography without intermediate information carriers (films, memory plates) is shown, providing a low cost and ability to multi-angle real-time monitoring of inner defects of welded joints. At the same time the speed of control is significantly reduced. The portable equipment for implementation of this technology is described allowing achieving the sensitivity of up to $0.1 \%$ of thickness of the inspected metal and resolution exceeding 10 pairs of lines per millimeter. 11 Ref., 4 Figures.
\end{abstract}

Key words: non-destructive testing, flash radiography, digital image, X-ray TV system, scintillator, CCD array, flat panel detector, inner defects of welded joints

The most widespread type of non-destructive testing (NDT) of quality of welded joints, materials and products is radiation control. It can be applied to the products of any material, geometry and thickness. Due to visibility of results the radiation methods are preferred both in the quality control of welded and brazed joints, as well as in control of casting quality. This method is used to evaluate the validity of other NDT methods.

In the recent years, the qualitative changes in the system of radiation NDT occurred, first of all due to appearance of new multi-element semiconductor detectors of radiation images, as well as intensive introduction of digital technologies for acquisition, processing and analysis of images. Using such detectors, the ionizing radiation, passing through the examined object and carrying information on its inner defects, is converted using the electronic means into array of electric signals, which are then digitized, processed and used to generate a digital image (DI) of the examined object. DI of the object contains information on its inner structure and is formed immediately during radiation, i.e. in real time. Such method of radiation control is called a digital radiography or a flash radiography [1]. This is actually a portable $\mathrm{X}$-ray television with electronic record of information, which may be posted in the Internet without its additional processing.
A distinctive feature of the flash radiography is absence of intermediate information carriers (radiographic films, semiconductor selenium plates, memory plates with photo-stimulated memory), which are now widely applied in welding industry. They require long operations of exposure, processing and special devices for reading of information. Respectively, the absence of such intermediate carriers allows significantly increasing the productivity and reducing the cost of quality control.

NDT based on portable X-ray TV technology, DI processing fundamentally changes the technology, increases spreading of the most valid radiation NDT. In the recent years the concepts «digital radiography» and DI found wide application. The hardware and software complexes of $\mathrm{X}$-ray films and their DI processing find ever wider spreading [2-6]. Now DI are obtained using three different technologies (Figures 1-3), the procedures of their processing are general, and this is an important trend in the modern radiation flaw detection. The most often DI are obtained by digitization of radiographs. Rarely they are obtained in processing of latent image, read from the memory plates of multiple use, or from digital detectors of the flash radiography.

DI, obtained by any of the three indicated methods, should be equally interpreted. The results of DI radiography processing as to their sensitivity and resolution should be no worse than the result obtained by negatoscope from radiographic film, i.e. the images on DI of the standard reference specimens should be identical to the images on X-ray films. 


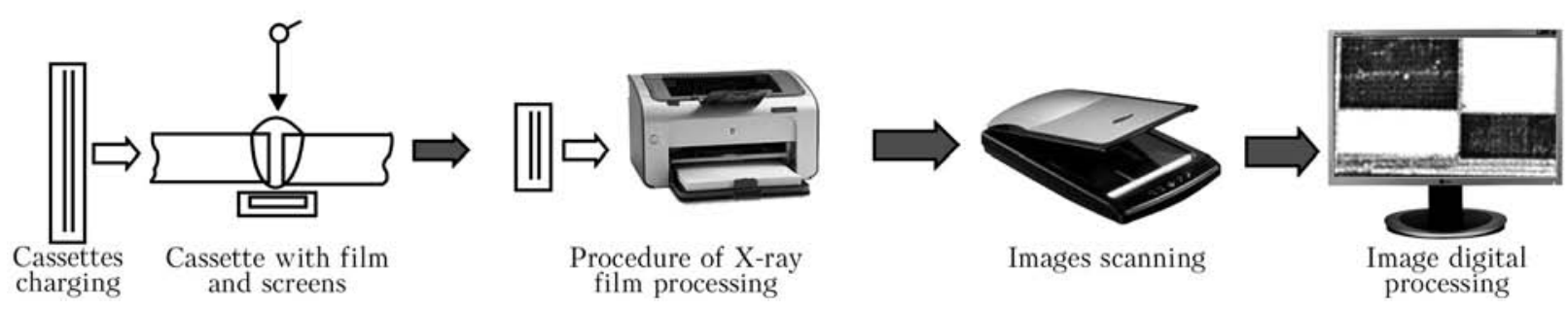

Figure 1. Traditional scheme of radiographic testing using film and digitizing of roentgenograms

The methods for obtaining DI of radiation control results in electronic form are different, but the principles of processing and subsequent decoding of these images are the same [1-7].

Figure 1 shows a classical technological scheme of obtaining DI due to digitizing of film radiographs. This technology is widespread throughout the world, requires preparation of cassete with film and screens. The radiation is followed by the procedures of chemical treatment, drying of the film, reading of information on negatoscope and digitization of results using the appropriate computer complex. This lengthy and expensive technology is used in all industrial sectors, mainly because of the possibility of a compact archiving of NDT in electronic form and for obtaining the additional information, which cannot be obtained without digitizing.

Figure 2 shows the scheme of a more advanced technology of obtaining DI based on memory plates. As compared to the previous scheme of obtaining DI, this technology provides possibility of multiple use of intermediate information carrier. In this case there is no wet process of developing. It provides quicker control, but does not make it cheaper and requires a higher qualification of personnel, much time on auxiliary operations and expensive reading equipment. Often memory plates have their own defects.

Highly evaluating the possibilities of obtaining the additional information during DI processing and an archaic nature of digitization of film images, the world film manufacturers, such as Agfa, Fudjie, Kodak and other took the way of replacement of film by semiconductor memory plates of multiple use.
Figure 3 shows the scheme of technology of instant (flash) digital radiography based on fluoroscopic and solid-state detectors [1-3]. This is the quickest and the cheapest way of obtaining DI of inner defects of welded joints, which does not require processing and reading equipment and the corresponding auxiliary time.

Both new types of filmless radiation control (see Figures 2 and 3) may provide better results than the results of the image digitization obtained using X-ray film.

The quality of X-ray image is determined by sensitivity standards (GOST 7512-82). The classification of welded joints according to the results of radiographic testing is carried out in accordance with GOST 23055-78. It is known that the higher density of blackening and the higher exposure, the more information an exposed film contains. Therefore, to digitize the films, to obtain their informative DI a good scanner is required. The widespread reading devices, inexpensive scanners cannot provide an acceptable quality of digitization of X-ray images if their relative density of blackening is higher than 3 . All the attempts to obtain a satisfactory DI from denser films were not successful. Therefore, in a variant with the film (see Figure 1) obtaining a satisfactory DI is possible if the optical density of the films is only in the range of 1.5-2.5. At such values the noises of digitizer do not introduce irreversible distortions into DI. The experience of digitization of film images with a density of 3.0-3.2 already shows unsatisfactory results, precise information is hardly reproduced. For example, the images of small pores of diameter less than $0.2 \mathrm{~mm}$ and cracks with small opening get

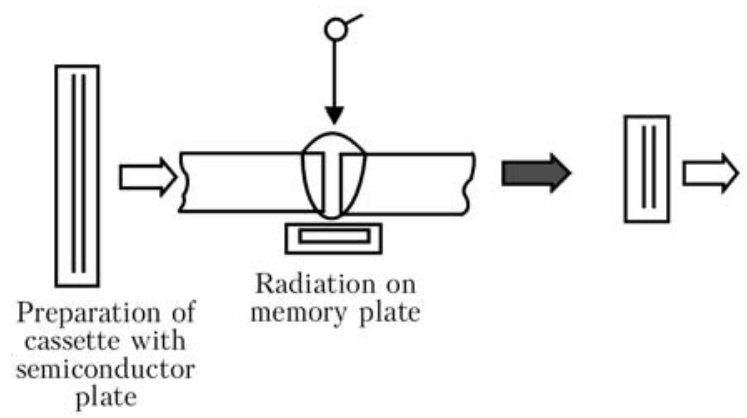

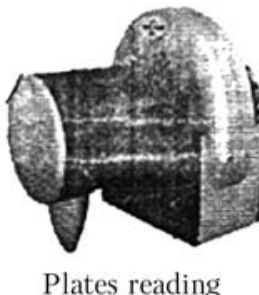

Plates reading

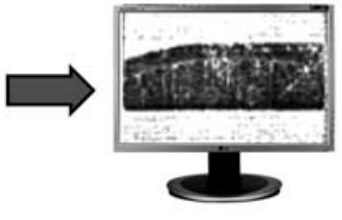

Processing and issue of conclusions

Figure 2. Scheme of radiation technology with the use of memory plate 
lost. Thus, digitization of films has significant limitations. A part of the defects detected using negatoscope is not found on DI. This is a significant drawback of traditional film radiography.

The filmless technologies according to the schemes of Figures 2 and 3 do not have this drawback, they differ in a large dynamic range, which extends the capabilities of NDT. The experience of DI analysis according to the technological schemes of Figures 2 and 3 confirmed that detection of small pores, cracks and inclusions in different welded joints exceeds the information on them on the film. The technology of flash radiography according to Figure 3 based on solidstate or optoelectronic transducers, where after the computer processing of DI it is possible to obtain the sensitivity of up to $0.1 \%$ and also to perform control in motion, has especially great prospects. Moreover, the detectability of defects increases due to the fact that the human eye distinguishes better the tiny moving images than those being in statics. In the absence of intermediate information carriers during radiation according to the scheme in Figure 3 there is a possibility to change its direction, i.e. the tomographic effect is present. For the three technologies (Figures 1-3) the obtained DI is easily archived and transferred via the Internet. The speed, cost of obtaining of one DI according to the technological schemes of Figures $1-3$ is approximately correlated as 10:5:1, and the cost of equipment for these purposes is respectively $5: 20: 1$.

Figure 4 shows the schematic diagrams of obtaining the DI of radiation control in electronic form according to three described technologies.

A common disadvantage of the first two techniques (Figures 1,2) with an intermediate information carrier is non-obviousness in correct designation of the primary radiation mode, the need in repeated, sometimes multiple radiation for finding the optimal values of anode voltage, exposure time, focal distance, as well as supporting procedures with the information carrier. Typically, for the operator to obtain a new object previously not subjected to testing, it is necessary to preset correctly the specified parameters most often by the repeated selection, i.e. with repetition of all the preparatory procedures before radiation. In case of the technology according to Figure 3 the parameters of radiation can be specified in the process of radiation during observation of changes in the image on monitor screen. Thus the optimal modes are found, the image of inner defect is studied from many angles of camera.

During the flash radiography [1] all three parameters or part of them $\left(U_{\mathrm{a}}, I, t\right)$ may vary,
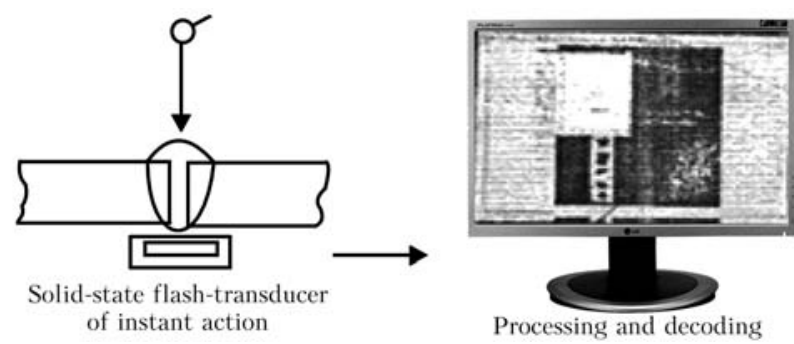

Figure 3. Scheme of radiation without intermediate information carriers

observing the changes of the image on the monitor screen in real time. This circumstance significantly reduces the time and consumables. In addition, the own artifacts of films, screens, memory plates, cassettes in the technologies with intermediate information carriers can be hardly deleted and is poorly defined. In case of studying the image in real time, i.e. according to the technology of Figure 3, with the possibility of varying the parameters of testing mode the artifacts of detector are easily detected and further corrected. The algorithms for work with electronic images are available, providing accumulation and subtraction of individual fragments in DI.

Study [2] of Prof. Majorov is devoted to the comparison of the image quality obtained from different detection systems. He notes that for the technologies without intermediate information carriers, the methods of calibration, subtraction of intrinsic noises of transducers were developed. Thus, they improve the contact sensitivity to such a level that it is possible to mark the changes of thickness radiation as $1 / 1000$, whereas using the best films of class $\mathrm{C} 1$ according to European Standard EN 584-1 and American ASTM E 1815 the best contrast of $1 / 100$ can be achieved.

The image quality [2] is characterized by many specific indicators:

- basic spatial resolution, measured using the reference with double wires (EN 462-5) equal to half of the registered smear or the effective size of pixel (in case of the technology according to Figure 3). The resolution power is determined by the distance of adjacent elements to the image. The value inverse to this distance is the spatial frequency measured in line pairs per one millimeter;

- smear of the image, which has a multifactorial origin due to geometry, projection circumstances, non-sharpness of the detector;

- signal/noise ratio (SNR), which depends on exposure and quality of radiation tract. This ratio increases as the square root of area of working pixels;

- contrast/noise ratio, which depends on SNR of the detector and absorption coefficient of the object material; 


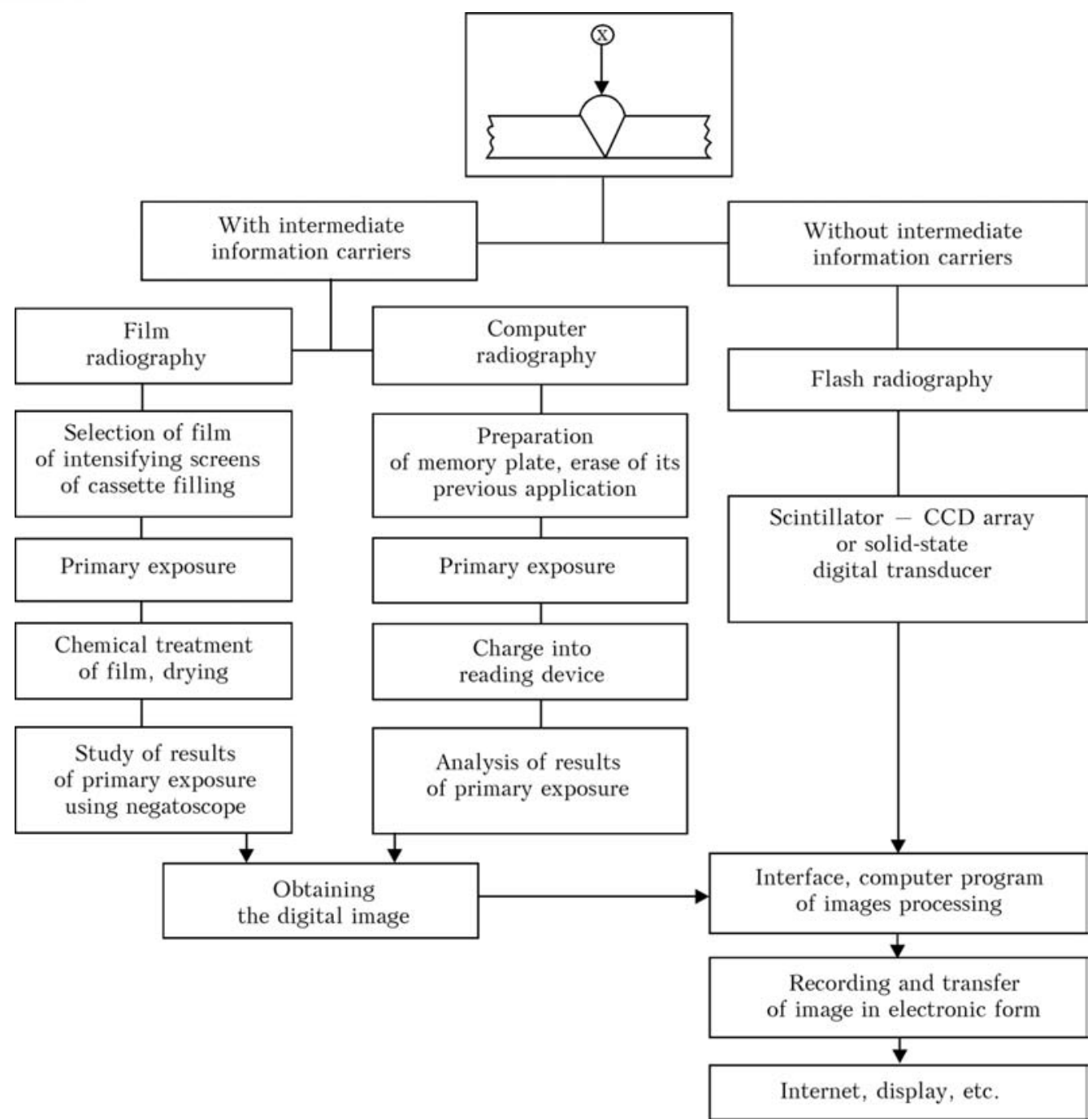

Figure 4. Schematic diagram of technological procedures of obtaining the results of radiation control in electronic form at film, computer and flash radiography

- dynamic range. This is the object thicknesses, available for a satisfactory analysis on one image. The large dynamic range provides significant advantages over the technologies according to schemes in Figures 2 and 3. Usually a large dynamic range is achieved due to the exposure dose, which in the film systems is limited by relative density of blackening 3-4. After that the films become unreadable. In case of digital detector systems (without intermediate carriers) due to the computer technologies «exposure», i.e. accumulation of information, has no limitations. SNR increases as the square root of the dose. This is equivalent to the exposure time or the number of averaged images. Thus SNR is achieved equal to several thousands and of high quality of DI. In practice, this process is limited to the contrast sensitivity of $0.1 \%$, which corresponds to SNR of about 1000.

Therefore, no doubt that the future belongs to the portable X-ray television without intermediate information carriers (memory plates, films, etc.) with the elements of scanning, changes in direction radiation passing through the object [8-11].

The schemes of digital processing of DI (see Figure 4), independently of their method of preparation are almost the same. They include operations of evaluating the fitness of DI, measurements of the intensity of grey, optical density and sensitivity. The digital scale of grey is 16-bit [6], has 65 thousand shades, and the histogram of DI must lie approximately at the center of the scale, to avoid underexposure or overexposure. The central location of the histogram provides digital processing of higher quality, i.e. allows scaling the intensity of grey. The calibration operation is applied according to the size, which allows measuring the defects, performing other procedures unusual for traditional film radiography.

\section{Conclusions}

1. The most informative one, with easy understandable results, is the radiographic method of 
testing welded joints. Using this method it is possible to achieve resolution of about 10 pairs of lines per millimeter, which is very important to detect different cracks in welded structures.

2. One of the serious disadvantages of film radiography is need in powerful light emitters (negatoscopes) in order to see the defects of object captured by X-ray film. Moreover, the darker the picture, the more information it contains on thin defects. At a relative density of blackening of more than 4 the pictures become almost unreadable. This drawback is absent in the modern filmless technologies, which provide, moreover, the result of radiation control in electronic form without special digitizing complexes.

3. The electronic digital information contains illustrative radiation images of inner defects, extends the possibilities of flaw detection, reduces the speed and the cost of control. This provides increase in both accuracy of control and its productivity. Especially the flash radiography is effective based on portable X-ray television. Moreover, intermediate information carriers (films, semiconductor plates) are not required, as far as the same sources of radiation are used, which are used in film radiography. It becomes possible to consider the inner defects from different camera angles, which is not available for other NDT methods.

4. The most challenging one is the flash radiography based on portable X-ray television technology with digital solid-state transducers. At the same time the sensitivity of up to $0.1 \%$ of the thickness of radiated metal and the resolution exceeding 10 pairs of lines per millimeter may be achieved. The use of small-sized movable solidstate transducers opens up new technological possibilities in NDT of quality of welded joints.

1. Troitsky, V.A. (2013) Flash radiography. Territoriya ND, Oct.-Dec., 44-49.

2. Majorov, A.A. (2009) Digital technologies in radiation control. V Mire Nerazrush. Kontrolya, 3, 21-24.

3. Troitsky, V.A., Mikhajlov, S.P., Pastovensky, R.A. et al. (2015) Modern systems of radiation nondestructive testing. Tekhn. Diagnostika $i$ Nerazrush. Kontrol, 1, 23-25.

4. Stepanov, A.V., Lozhkova, D.S., Kosarina, E.S. (2010) Computer radiography of results of practical investigations of possibility to replace the film technologies, 11-14. Moscow: VIAM.

5. Grudsky, A.Ya., Velichko, V.Ya. (2011) Digitizing of radiograms is not very simple. V Mire Nerazrush. Kontrolya, 4, 32-34.

6. Tsvetkova, N.K., Novitskaya, K.A., Kologov, A.V. et al. (2014) Peculiarities of application of digital radiography complexes in nondestructive testing of casing production. Tekhnologiya Mashinostroeniya, 7, 47-50.

7. Varlamov, A.N. (2014) Experience of operation of digital radiography complex in field conditions. $V$ Mire Nerazrush. Kontrolya, 63, 25-28.

8. Majorov, A.A. (2007) X-ray television in industrial NDT. Ibid., 35(1), 4-9.

9. Zscherpel, U., Ewert, U., Bavendiek, K. (2007) Possibilities and limits of digital industrial radiology: The new high contrast sensitivity technique - Examples and system theoretical analysis. Lyon.

10. GOST 27947-88: Nondestructive testing. X-ray TV method. General requirements. Moscow.

11. GOST 29025-91: X-ray TV detectors. General technical requirements. Moscow.

Received 09.04.2015 